\title{
DETECTION AND PURIFICATION OF HARMFUL COMPOUNDS IN BEVERAGES USING HPLC
}

\author{
Rukkumani, $\mathrm{V}^{1^{*}}$, Saravanakumar, $\mathrm{M}^{2}$ \\ Assistant Professor (Sr.G) , Department of Electronics And Instrumentation , Sri Ramakrishna Engineering \\ College, Coimbatore \\ Assistant Professor, Department of Physics, SVS College of Engineering, Coimbatore \\ rukkumnai.v@srec.ac.in
}

\section{ABSTRACT}

The presence of harmful compounds like caffeine and carbonated compounds in different beverages like soft drinks, fruit juices deserves great attention because of its toxic and carcinogenic effects on human beings. We report on the detection and purification of those substances with the help of HPLC(High Performance Liquid Chromatography).According to the migration rate, stationary phase and mobile phase, retention time we can extract the desired compounds. Depending upon the solvent and sample we can detect the compounds with the help of the detector. The chromatogram will be displayed and it can be viewed in the PC with the help of Osiris software. Compounds like Caffeine, Aspartame, Neotame, Saccharin, Maltodextrin, sucrose, fructose etc can be detected and purified. Detection and purification takes place in the column of HPLC where the process called adsorption takes place. Retention time can be calculated by the total time taken of a component that spends in both mobile phase and stationary phase. It is always expressed in minutes.

Keywords: Retention Time, Stationary Phase, Mobile Phase, Adsorption, Migration Rate

\section{Academic Discipline And Sub-Disciplines}

Engineering , Physical sciences

\section{SUBJECT CLASSIFICATION}

Materials and Chemical Properties

\section{INTRODUCTION}

Beverages are consumed by many people all over the world. Nobody knows the exact ingredients of the product except the manufacturer. These beverages have a fatal effect on the human beings. People suffer a lot by consuming this which consists of many harmful compounds. So, our project reports on the presence of harmful compounds and its composition. By doing this we can find the real ingredients present in the product. The ingredients present in the beverages may cause many harmful effects like increase in blood pressure, hypertension, insomnia, headaches, diabetics and allergies.

\section{EXPERIMENTAL SETUP}

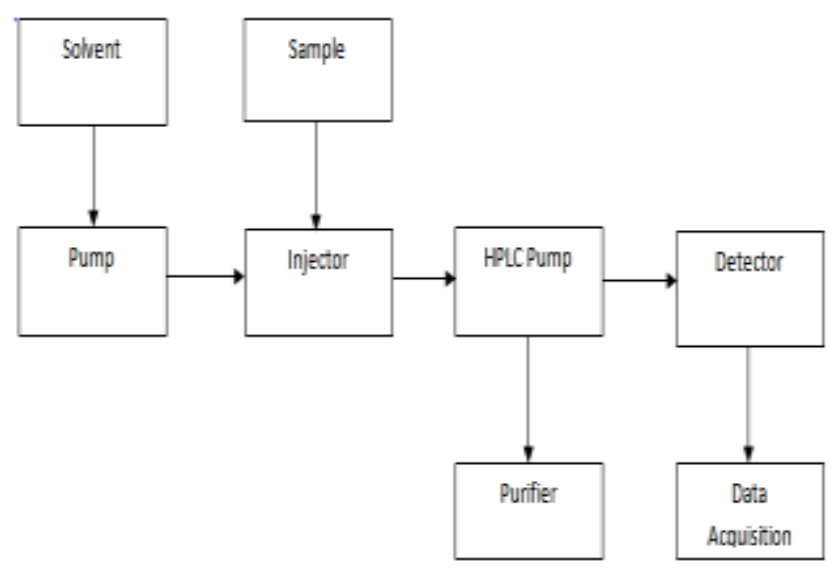

Fig.2.1 Block Diagram of Detection and Purification of Harmful compounds in beverages using HPLC.

\subsection{Explanation}

The block diagram of Detection and Purification of Harmful compounds in beverages using HPLC describes the working of the HPLC Column, Injector, reciprocating pumps and the detectors employed. 


\subsection{Solvent}

The solvents used in HPLC are Methyl Butyl Ether, Methanol, Isobutyl Alcohol, Hexanes (95\% n-hexane), Heptanes, Ethyl Acetate, Ether, Dichlorobenzene, Cyclohexane, Chloroform, Hydrocarbon Stabilized, Acetonitrile 2-Propanol, Toluene and Water[1].

\subsection{Pumps}

The purpose of the solvent delivery system (Pump) is to deliver a continuous pulse free flow of mobile phase to the HPLC system - regardless of the system back pressure. An ideal pump should have the following desirable characteristics are Solvent compatibility and resistance to corrosion Constant flow delivery independent of back pressure Convenience of replacement of worn out parts Low dead volume for minimum problems on solvent changeover.

\subsection{Sample Pretreatment Methods}

Pretreatment before HPLC analysis is often required for samples of low concentration or samples containing analytical contaminants[3]. It improves reproducibility and sensitivity in analysis, and protects HPLC columns. The pretreatment methods are different according to the each sample. Filtration is a method of pretreatment. It is a common method used for separating solids from liquids. It extends a column's life by minimizing column damages from solid contaminants such as particles, sediments and colloid substances. It also improves reproducibility of analytical data.[4].

\subsection{Injectors}

Injectors for liquid chromatographic systems should provide the possibility of injecting the liquid sample within the range of 0.1 to $100 \mathrm{ml}$ of volume with high reproducibility and under high pressure (up to the $4000 \mathrm{psi}$ ).[5].

\subsection{Detector}

A chromatography detector is a device used in gas chromatography (GC) or liquid chromatography (LC) to detect components of the mixture being eluted off the chromatography column [6]. There are two general types of detectors: destructive and non-destructive. The destructive detectors perform continuous transformation of the column effluent (burning, evaporation or mixing with reagents) with subsequent measurement of some physical property of the resulting material (plasma, aerosol or reaction mixture). The non-destructive detectors are directly measuring some property of the column effluent (for example UV absorption) and thus affords for the further analytic recovery[7].

\section{RESULT AND DISCUSSION}

The output peaks of the compounds such as Caffeine, Sucrose, Aspartame, Fructose, Neotame are obtained as shown in Fig.1.2. The compound caffeine is found to have the highest retention time. The output is obtained using the Orisis software tool.

Depending upon the Retention time, the compounds are obtained in the order as shown in Table 1. The amount of sample injected varies depending on the type of standards used. From the compounds detected, the chemical compounds are classified and an analysis was made such that caffeine has the highest retention time followed by fructose, neotame, aspartame and sucrose which are the sweetening agents added to the beverages.

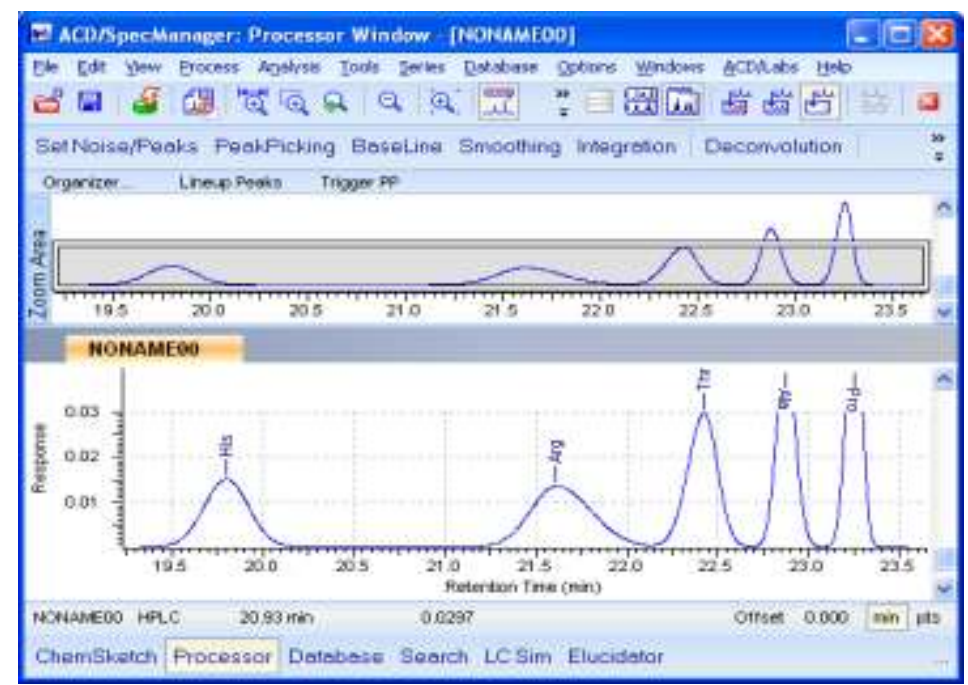

Fig.1.2 Output peak of the compounds

5216 | P a g e 
Table :1 Output results of the compounds

\begin{tabular}{|c|c|c|}
\hline $\begin{array}{c}\text { Chemical } \\
\text { Compounds }\end{array}$ & $\begin{array}{c}\text { Injected } \\
\text { Sample }\end{array}$ & Retention Time \\
\hline Sucrose & 0.1 & 19.8 \\
\hline Neotame & 0.1 & 21.6 \\
\hline Aspartame & 0.35 & 22.4 \\
\hline Fructose & 0.4 & 22.8 \\
\hline Caffeine & 0.43 & 23.25 \\
\hline
\end{tabular}

Form this result, the detection and purification of some harmful compounds such as caffeine, neotame, aspartame, sucrose and fructose was done. Also the analysis of the composition of chemicals in the beverages that is consumed in our day to day life was done.

In future, UHPLC(Ultra High Performance Liquid chromatography) column for the process can be used so that more number of compounds and its chemical composition can be detected. Accuracy and resolution can be improved by the use of UHPLC columns.

\section{REFERENCES}

1. Bauersfeld, M.L. and Wollenstein, J. (2011) 'Miniaturised gas chromatographic system with metal oxide gas sensor array for fast detection of off flavours', International Journal of Students in Technology and Management, Vol.12, No.8, pp.2086-2089.

2. Braunrath, R. (2005) 'Determination of bisphenol A in canned foods by immunoaffinity chromatography, HPLC, and fluorescence detection ', Journal of Agriculture, Vol.53, No.23, pp.8911-8917.

3. Fang , F . (2007) 'Determination of red wine flavonoids by HPLC and effect of aging', Journal of food chemistry, Vol.101, No.11, pp.428-433.

4. Domenico Caputo, S. (2012) 'Amorphous Sillicon Photosensors for Detection of Ochratoxin A in Wine' , International Journal of Students Research in Technology and Management, Vol.18, No.6, pp.2674-2677.

5. Irwandi Jaswir, K. and Rini Akmeliawati, M. (2013) 'IlUM-Fabricated portable electronic nose for Halal authentication in beverages', IEEE Transactions, Vol.11, No.13, pp.978-981.

6. Jolliffe, I.T. (2006) 'Principal Component Analysis', IEEE Instrumentation and Measurement Magazine, Vol.18, No.5, pp.2145-2150.

7. Yoshioka N (2008) 'Determination of 40 synthetic food colors in drinks and candies by HPLC using a short column with photodiode array detection', Journal

of food chemistry, Vol.74, No.5, pp.1408-1413.

8. Ming Ma, Xubiao Luo (2006) 'Simultaneous determination of water soluble and fat soluble synthetic colorants in foodstuff by HPLC', Journal of chromatography, Vol.1103, No.19, pp.170-176.

9. Juan V. (2009) 'Determination of Melamine in milk based products by ion pair liquid chromatography-tandem mass spectrometry', IEEE Instrumentation and Measurement Magazine, Vol.649, No.17, pp.91-97.

10. Zhao Yingbo, S. and Zhou Yanming, S. (2011)' Determination of gibberellin residue in vegetables and fruits by HPLC-MS/MS method', IEEE Instrumentation and Measurement Magazine, Vol.11, No.8, pp.3981-3985.

\section{Author' biography with Photo}

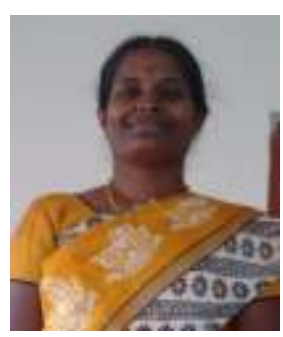

Dr.V.Rukkumani , ME, Ph.D, working as a Assistant Professor (SG) in Sri Ramakrishna Engineering College, Coimbatore. She has Completed Doctorate degree in the year 2015 in Anna University, Chennai with 7 International publications. She also received Dr.A.P.J Abdulkalam Award for her teaching service. 